\title{
Structure and Mechanical Properties of Soft Tissues during Selected Pathological Processes
}

\author{
Barbara Kmiecik $^{1^{*}}$, Anna Skotny ${ }^{2}$ and Jerzy Detyna ${ }^{1}$ \\ ${ }^{1}$ Department of Mechanics, Materials Science and Engineering, Wroclaw University of Science and Technology, Wrocław, Poland \\ ${ }^{2}$ Department of Internal Diseases, Geriatrics and Allergology, Medical University of Wroclaw, Wrocław, Poland
}

"Corresponding author: Barbara Kmiecik, Department of Mechanics, Materials Science and Engineering, Wroclaw University of Science and Technology, Wrocław, Poland, Tel:+48 695534293 ; E-mail: barbara.kmiecik@pwr.edu.pl

Received date: January 20, 2017; Accepted date: February 18, 2017; Published date: February 24, 2017

Copyright: (c) 2017 Kmiecik B. This is an open-access article distributed under the terms of the Creative Commons Attribution License, which permits unrestricted use, distribution, and reproduction in any medium, provided the original author and source are credited.

\begin{abstract}
Pathological process often modifies the structure of the soft tissue and can lead changes of mechanical properties. The regularity of this relationship has already been used in the elastography and currently it is applied in medical diagnostic. Tissue stiffness can be identified by a quick measurement but more accurate details can be obtained only by biopsy. Studies, presented in a scientific literature, focus only on pathological stiffness or consider only changes in biochemical structure. These researches revealed two very important components of those modifications - elastin and collagen. Soft tissues are multicomponent, inhomogeneous and anisotropic structures. Their functionality depends on complicated processes on molecular level. Probably the individual components of the tissue can effect on each other. They may promote creation of processes, which can participate in modifications of elasticity. For this reason, it is very important to relate changes on the structural level with mechanical properties. This information can be very helpful in diagnosis and treatment of soft tissue disease.
\end{abstract}

Keywords: Soft tissue; Elasticity; Collagen; Pathology

\section{Introduction}

The same components can create different tissue in human body. Mayers and Chen in their work were trying to extract the most important elements of the various biological systems. Three main components of human body are water (58\%), lipids (17\%) and proteins (15\%) [1].

All mentioned above biological elements probably determine mechanical properties of the tissue. The rest of components can probably influence on these structures condition but they have no direct relation with the biological elasticity of the tissue.

Cells are the most elementary element of our body. Each tissue is built of the same components but their concentration is strongly associated with the function of the tissue. Modification at the molecular level are visible when changed area is bigger than the resolution of medical diagnostic devices. (ultrasound, NMR, TK). So far the only sign of change at the molecular level is the modification of tissue stiffness. Paper of Moeendarbary and Harris have presented effects of the influence of selected factors on the smallest part of the organism. Authors consider the cell as a separate phase material, which consist $60 \%$ of water. They suggested that this component acts as a determining factor of the cytoskeleton behavior [2]. This assumption is not new, it had been used in earlier publications, where interactions of water and proteins were analyzed [3,4]. Moeendarbary and Harris recall a two-phase nature of the cytoplasm. This structure is composed of a cytoskeleton, organelles and macromolecules, which are present in intracellular fluid-cytosol. According to this model, cytoplasm is a flexible and durable mesh which is sensitive to pressure changes inside the cell. Viscoelastic properties of the biological cell depends on the flow of the not-cellular fluid in response to mechanical impact [2].
An additional aspect is that surrounding structures can influence on mechanical properties of soft tissue [5]. This means that the nature of the analyzed biological structure depends on the condition of bones tissue and muscles. Furthermore, these elements are susceptible to various factors (such as temperature or pressure of the fluids), which can modify their state. These observations may explain why it is so difficult to define the characteristic features of the tissue. Constant changes in the human body also can modify mechanical properties due to their excellent adaptation to the local environments. For this reason, determination of biological tissues features should be performed by invivo measurements. However, the definition of changes in the tissue, under the influence of external factors, can be extremely difficult and invasive if those studies would be held in the human body. So far, the scientists determine a few of skin elements which can influence on the structure and mechanical properties. The most important are proteins (collagen, elastin, keratin), water and lipids whose concentration and conformation can regulate the stiffness and the structure of the tissue.

\section{Skin-protective layer}

Skin is the most widely studied and described connective tissue in the human body. Due to the presence of protective properties and extraordinary abilities it has been incorporated into the ranks of smart materials. Skin and its epidermal layer play an irreplaceable role in the body. Regulation of the water is one of the most important function $[4,6,7]$. Life without this substance is impossible.

In the human body water is present in two different conformations. A bounded form is when hydrogen is bonded to different functional groups. Water can be also in a free state. In that case, molecules of this substance are no hydrogen bounded to other biomolecules. Concentration of these forms is determined by structural properties and performed functions of the tissue. Water in biological systems is combined with other molecules through hydrogen bonds. Many 
studies were carried out and it has been proved that in the skin, water is mostly present in the bounded form [8]. This can means that aqua molecules in the free form are strongly connected to each other and they are able to form a separate water pools inside the tissue. It has been suggested that it is used in certain processes and provide continuous work of biological processes.

In conditions of low humidity molecules of water are strongly connected to keratin polar groups. In opposite case, a lot of places in this protein are exposed to the outside environment. It may lead to creation of additional bonds with water and its accumulation [9]. A study carried out by Bouwstra suggest that the keratin domain are not present with a high water content. Probably those molecules can be captured by corneocytes earlier [10]. In conditions of high humidity water is accumulated in the extracellular area in small pools $[10,11]$. Probably this process minimizes the contact area between the hydrophilic and lipophilic lipid domains [10]. Those data suggest that water can react with proteins and lipids, which is compatible with the general construction of the skin [12].

$\mathrm{Wu}$ and van Osdol in their paper analyzed influence of selected factors on the structure of the most external layer of the skin - stratum corneum (S.C.). They suggested that water may have a negative impact on intercellular corneocytes. The excess of this substance can lead to lipid liquefaction. Simultaneously, researchers claim that the rate of lipid conformations changes is very small and can be neglected. Authors also suggest that temperature has the most significant role for lipids structure [13]. Increasing of this factor lead to reorganization of lipids conformation from ordered to disordered [14]. This process is probably linked to raise water permeability due to the temperature growth. However, as the result of arising phase changes, significant energy shifts has not been identified. This leads to the suggestion that intercellular structure does not be very susceptible to changes in lipids order. However, performed studied indicate that their presence is necessary to maintain the diffusion process. Lipids also play an important role in the amortization of impact of mechanical factors on the skin. Young's module (YM) single cell of S.C. is bigger than YM for the whole tissue. It may confirm the important role of bonds between water and lipid molecules. Probably they determine the elasticity of the skin. It has been suggested that abnormal lipids conformations can cause some skin pathology [13].

Extraordinary mechanical strength of hair and nails is associated with a specific order of proteins structure. This attribute is determined by the specific construction of those biopolymers. Chemical and mechanical strength of proteins is enhanced by multiplied amount of disulfide bridges. S-S bonds present in hair and nails are very stable due to the gausche-gausche conformation in their structure. Analysis of albumin and phosphate acid isoenzymes suggests the relationship between the quantity of S-S and gausche-gausche bonds with the resistance to proteins denaturation [11].

Skin is a connective tissue, with a high concentration of protein. It is composed mainly of collagen (type I and III) [15], elastin and hyaluronic acid. These proteins play an irreplaceable role in all biological structures present in the human body. Collagen and elastin are responsible respectively for stiffness and flexibility. Shape of these biopolymers is linked to biochemical properties. Changes of it may lead to functions modification. An example of such process is a hyperplastic scar tissue which can arise as a result of abnormal wound healing [16].
In 2002, scientists published a paper, where they suggest that observed differences in skin color and pathologies are not caused only by variations in the genetic code. Probably behaviour of individual ethnic cultures can influence on skin condition [17]. These results confirm the impact of external factors on the structure and processes in tissues.

\section{Pathological processes of the skin and the distribution of water in the skin}

Probably, poor water permeability through epidermal layer is the most common cause of skin disease. Malfunction of this structure probably is associated to excessive proliferation and leads to uncontrolled exchange of stratum corneum [18].

Recent studies suggest that dermatological changes (stretch marks, excessive xerosis) may accompany some diseases. It can be manifested by increased body weight, which probably is caused by low water permeability in the skin [7]. Skin lesions associated to disorders of the metabolism also refers to children. However studies done by Nino suggested that in the case of juvenile persons, dermatological problems are correlate only with the most sick patients. One of that modification is called plantar keratoses, and it occurs as the result of excessive pressure on the foot surface. Researchers also recalled a study of speed of transepidermal water loss by obese organisms. They suggested that children with the high level of the fat, probably overheat easier, which can lead to increased activity of sweat glands. The result of this process probably Friction, capacitance and transepidermal that processes described above probably involve other structures presence. Main substances which can influence on water metabolism are adipocytes and leptins. Researchers think that fat cells mentioned above, promote the process which can cause the replacement of the stratum corneum and increase the activation of keratinocytes. It is suspected that leptin supports the proliferation of fibroblasts and collagen synthesis $[19,20]$.

Water permeability is not a problem only in metabolism diseases. The inflammatory reaction of epidermal layer can also be related with it. Eczema, psoriasis or ichthyosis are very good examples of this irregularity. These illnesses are connected to modified order of lipids in the epidermal layer. These differences affect the economy of water and correct skin irrigation is impossible. In atopic dermatitis, invalid biosynthesis of ceramides and acylceramides causes a disturbance in the body's barrier function. In this tissue, lamellar body are placed in the higher layers than in healthy one. A similar situation occurs in the case of psoriasis, where increased amount of parakeratotic corneocytes [18] and loss intraepidermal water is observed [21]. The fundamental problem in ichthyosis is drastically decreased levels of the endogenous $\beta$-glucocerebrosidase enzyme, responsible for the ceramides hydrolysis. This situation leads to lipids architecture modification, which is noticeable in diseases [18]. These abnormalities can cause the malfunctioning of the epidermal layer and incorrect irrigation of the skin. Reduced amount of hydrogen bond lead to increased tissue stiffness and it may provoke increased susceptibility to damage and injuries. In addition, it will most likely to be an easy target for infections, due to the incorrect protection function.

Modification of skin mechanical properties during pathological processes has not been fully understood. However, many researches focused on different mechanical and biochemical features of young and old people skin tissue. Organism aging causes reduced elasticity of the soft tissue [22]. This process occurs probably as a result of structural changes in proteins and their hydration. Effects of that 
action are visible as morphological changes of the skin - wrinkles. Organism ageing leads to chemical bonds modification in proteins and water [11]. Skin becomes more slim, stiff and it is no longer so tense [23]. The existing research agrees that the lipid structures help in preventing water loss and penetration in substances readily soluble. As a result, it can be suggested that mentioned compounds are responsible for skin elasticity and make everything is packed so tightly as it is possible [24]. All of the processes mentioned above lead to increase of Young modulus of the tissue over the time $[1,15]$.

\section{Invalid collagen synthesis}

Hyperplastic scars are slightly extended structures on the skin surface, which can appear as a result of inappropriate wounds healing. This defect can be characterized by a red or pink tint but it is not larger than the original size of the injury. In the healthy skin, collagen bundles are arranged parallel to the surface of the epithelium. In the scarring process, bundles of collagen type III are arranged in a distinctive and undulating manner. This situation occurs when function of proteins responsible for regeneration is impaired. During the healing process, collagen synthesis and degradation of unnecessary biological structures are damaged. Hyperplastic scarring lead to increase of collagen content in tissue, and it can be even 20 times higher in comparison with the healthy tissue $[15,25,26]$.Other studies suggest that in formation of hyperplastic scars may take part also arachidonic acid [27], as evidenced by involvement of lipids in that process. Hyperplastic scars, because its structure is stiffer than healthy tissue [16]. Some scientists try to combine these scars and disease leading to kidney or liver tissue fibrosis [28].

\section{Cancer changes}

Several pathological processes modify soft tissue structure and lead to very serious diseases like cancers. Stiffness of malignant tumour is bigger compared with healthy tissue and this feature let to distinguish them from a fundamental tissue of the organ [26]. Extracellular matrix with collagen fibers is a probably the most important content of the carcinoma and it may be the result of a specific structure of this cell formation. Accumulation of cancer altered cells leads to local changes in tissue stiffness. If the size of this modification is greater than the resolution capabilities of diagnostic equipment, it will be identified. The most of these devices analyze biological tissue stiffness. Proteins are the main component of the cytoskeleton and they are created in the same place where the extracellular matrix. All these biological elements combine with each other and form a tissue. Modification of structural and biochemical properties of cytoskeleton and extracellular matrix can be the first stage of cancer development. Suresh suggest that secreted proteins affect the mechanical capacity of these biological structures and remodel their ability to compression or stretching. As the result of mentioned processes, cancer altered cells improve their movement and can migrate to the most distant corners of the body and create metastasis [29].

The structure of the cytoskeleton is very complicated with three cytoskeletal elements like microtubules, actin and intermediate filaments. Each of these elements are responsible for strength and elasticity of the cell. Microtubules are resistant to compression and with the actin can influence on cell stability. All those three cytoskeleton elements take part in a response to the impact of the environment on the cell, and each of those structure act a different role in this process. It is possible due to their unique mechanical properties (Table1) [29]. Same publications can prove the relationship of deformability and actin content. The tissue is becoming more elastic, when the amount of actin was decreasing [30].

\begin{tabular}{|l|l|l|l|l|}
\hline Biological structure & Diameter $[\mathrm{mm}]$ & Bending stiffness $\left[\mathrm{Nm}^{2}\right]$ & Young's Modulus $[\mathrm{GPa}]$ & Length $[\mu \mathrm{m}]$ \\
\hline Microtubules & $14-25$ & $2.6 \times 10^{-23}$ & 1.9 & 6 \\
\hline Actin & 10 & $4.0-12.0 \times 10^{-27}$ & $1-5$ & $1-3$ \\
\hline Intermediate filaments & $6-8$ & $7.0 \times 10^{-26}$ & $1.3-2.5$ & 15 \\
\hline
\end{tabular}

Table 1:Mechanical parameters of selected biological structures [25].

\section{The tissue of the heart}

Collagen, an elastin and proteoglycans play the most important roles in the heart tissue. Unfortunately there is only a few researches which can connect molecular structure and mechanical properties of the heart tissue. Fomovsky suggests, that collagen play one of the most important roles in heart tissue function [31]. In spite of its small amount, this protein has a big impact on mechanical properties of this blood pomp. During the heart contraction, the biggest stresses go through the wall. However, surrounding elements like collagen fibers and other ingredients, compensate this stress which have place in myocytes. In diastole, collagen fibers become straight and the heart chamber fills with blood. This process probably protects cells of the heart from excessive stretching. Research also analyzed stiffness and content of collagen in heart tissue, what let suggests that in pathological changes several factors affect this blood pomp at the same time. One of the most dangerous phenomena is a heart attack and it can unprofitably change collagen content, structure and their location. Scarring of the heart muscle deserves for a special attention. It can be created after dead of a myocardium cell. Orientation of collagen and its content is changed in that situation. This is one of the reason why scientists try to determine relationship between this protein and heart mechanics [31,32].

The second very important ingredient of myocardium is an elastin. However, its role in the heart is unknown. Probably diseases which can lead to changes in collagen content and structure also influence on elastin. However, it is not clear how it will effect on mechanics of the heart. Role of elastin in arteries is very important. Analyzed protein is responsible for aorta stretching. Elastin provides constant perfusion during normal blood flow. However, in the case of higher pressures, collagen fibers are able to transmit compressive loads. Subsequently it gradually straightens and limit the expansion of the arteries. Fomovsky also recall the research suggesting that with increasing the distance 
from the centre (the heart) the elastin/collagen ratio changes in favour of collagen. This situation is associated with an increase stiffness of arteries. A similar mechanism occurs in the process of aging In that case, stiffening of the arteries is caused by the fragmentation of elastin and collagen deposition [31].

Proteoglycans are also a very important component of the myocardium. Many studies suggest that they can regulate the flow of water from the tissue. However, nobody can confirm that proteoglycans can play a similar role in the myocardium. Increasing attention is drawn to the smooth movement of the heart, which can be controlled by proteoglycans [31].

Chaturved suggests that stiffness of pressure-overloaded myocardial tissue is greater compared with healthy heart [33]. This may mean that under the increased stress on the heart wall, structure of the tissue can be changed. It can lead to increased stiffness of the myocardium. This modification is probably cause by collagen, which can probably change conformation and lead to increase of the stiffness in a heart tissue.

\section{The use of tissue elasticity changes in medical diagnosis}

The liver is one of the most important organs in human body. During the pathological process, this structure changes as well as its mechanical properties. The results obtained over the number of years have shown that the stage of liver fibrosis is strongly correlated with the elasticity. Elastic modulus of fresh human liver ranges from 590-1730 Pa at various stages of fibrosis. Mean liver stiffness increases with the degree of liver fibrosis (grades 1 and 2, however, are virtually unnoticeable) [34-36].

Cancers of the breast are very dangerous, and at the same time becoming more and more common diseases. However it can be diagnose very quickly due to the location. Cancerous tissue is stiffer than the fat and the glandular. In addition, it has more non-linear stiffness increase compared with other tissues (approximately 10:1 to 50:1). A study done by Wellman has shown that there is a significant correlation between the stiffness and histological structure. Such a phenomenon can be very useful in the diagnosis and it can lead to a faster diagnosis for some disease [37].

$\mathrm{Li}$ and Liu presented the device for diagnosis of soft tissue. Presented system allows to detect changes in the stiffness of the tissue. They performed in-vitro studies, which showed how easy pathological modification can be determinate. Li and Liu also presented how quickly and low cost diagnosis can be [26].

\section{Discussion}

The information presented above clearly suggests that pathological processes change structure of the tissue and probably can lead to mechanical properties modification. As a result, the tissue becomes stiffer, which usually lead to the impaired work of the biological structure. However, modification of the connective tissue, depends of the cause and it location.

Elasticity of tissue can be determined by hydration and proteins conformation, in particular the collagen. Behaviour of this biological structure can be determined by hydrogen bonds. It can connect water to proteins or lipids and change collagen biochemical structure. It has been suggested that mechanical and biochemical factors probably influence on hydrogen bonds and lead to their destruction. Conformational changes of proteins and lipids, can be observed in pathological states and may be cause by hydrogen bonds modification.
For example, incorrect lipids organization in skin diseases leads to impaired water exchange. As a result, the skin becomes stiffer and less resistant to external factors.

Presented information suggests that pathological processes also can lead to structural modifications. Malfunction of the tissue can be caused by an abnormal synthesis which can lead to incorrect proportion between components of the tissue. Skin changed by hyperplastic scar tissue contains more collagen type I than collagen type III [38]. A similar situation occurs in the case of liver tissue fibrosis. Pathological processes in production of proteins probably leads to liver cirrhosis [39]. Those situations can be associate to impaired degradation of collagen type I. Conformational changes of this protein also occur in people with heart failure. As a result of proteins modification, increasing of collagen type I content is observed [40], What lead to change of tissue stiffness? It is worth mentioning that in arterial vessels, protein structure also can be altered during pathological processes. The above considerations allow to conclude that stiffness tissue modification are not one-way process. In the case of the heart, the liver or even abnormal wound healing, the impaired function of the degradation can be the reason [38-40]. However, skin diseases modifications of lipid conformations can be the source of problems. Unfortunately formation of the cancer is unexplained process. These structures are stiffer than the surrounding tissue and can be quickly identify. The etiology of processes leading to such effects has not been clarified. It is suggested that impairment collagen degradation lead to formation of intercellular matrix. Probably the cancer altered cells compress surrounding biological elements and induce changes in those structures. In this way it is created an area of tissue, with modified properties. In all mentioned diseases, abnormalities in the processes at the cellular level lead to structural changes which can start of tissue elasticity modification. There is also the probability that modifications of the mechanical properties may depend on processes at the cellular level.

The biggest changes of young's module can be connected to modification of the content and the structure of collagen. Whereas the smaller ones may be related to the modification of conformations of lipids conformation. If this assumption will be confirmed, then it would be useful to design a method which allows distinguishing these two processes. It could speed up diagnosis of diseases, whose etiology can be related to impaired collagen degradation and synthesis.

\section{Conclusion}

Presented review suggests that modifications of tissues mechanical properties can be the result of changes at the molecular level. Probably the size of those changes can depend on the preceding processes. Frequently, these changes can be the result of the adaptation of the organ/tissue to pathologically environment. For this reason, it can be suggested that these modifications will probably be specific to an adequate disease. It is recommended to continue the study on mechanical properties of pathologically changed areas of soft tissue, which could improve existing measurement methods. If these assumptions would be correct, it would be possible to diagnose some conditions (associated with a change in tissue stiffness) very quickly and the treatment could be started immediate. 


\section{References}

1. Meyers MA, Chen PY, Lin AYM, Seki Y (2008) Biological materials: structure and mechanical properties. Progress in Materials Science 53 : 1-206.

2. Moeendarbary E, Harris AR (2014) Cell mechanics: principles, practices, and prospects. Wiley Interdiscip Rev Syst Biol Med 6: 371-388.

3. Xinxin GUO, Robert E, IMHOF J de R (2001) Spectroscopic study of water-keratin interactions in Stratum Corneum. Analytical Sciences/ Supplements. 17: 342-345.

4. Joachim W Fluhr, Peter E, Enzo Berardesca HIM (2004) Bioengineering of the skin: Water and the Stratum Corneum, 2nd Edition.

5. Flynn C, Stavness I, Lloyd J, Fels S (2015) A finite element model of the face including an orthotropic skin model under in vivo tension. Computer methods in biomechanics and biomedical engineering. Taylor \& Francis 18: 571-582.

6. Madison KC (2003) Barrier Function of the Skin: "La Raison d'Être" of the Epidermis. J Invest Dermatol 121: 231-241.

7. Guida B, Nino M, Perrino N, Laccetti R, Trio R, et al. (2010) The impact of obesity on skin disease and epidermal permeability barrier status. Blackwell Publishing Ltd 24: 191-195.

8. Gniadecka M, Nielsen O (1998) Structure of water, proteins, and lipids in intact human skin, hair, and nail. J Invest Dermatol110: 393-398.

9. Kasting GB, Barai ND (2003) Equilibrium water sorption in human stratum corneum. 92: 1624-1631.

10. Bouwstra JA, de Graaff A, Gooris GS, Nijsse J, Wiechers JW, et al. (2003) Water distribution and related morphology in human stratum corneum at different hydration levels. J Invest Dermatol 120: 750-758.

11. Gniadecka M, Nielsen OF, Wessel S, Heidenheim M, Christensen DH, et al. (2010) Water and protein structure in photoaged and chronically aged skin. J Investigative Dermatology 111: 1129-1133.

12. Mathur V (2010) Physical and chemical penetration enhancers in transdermal drug delivery system. Asian Journal of Pharmaceutics 4: 173-183.

13. Wu KS, van Osdol WW, Dauskardt RH (2006) Mechanical properties of human stratum corneum: Effects of temperature, hydration, and chemical treatment. Biomaterials. 27: 785-795.

14. Moore D, Rerek M (2000) Insights into the molecular organization of lipids in the skin barrier from infrared spectroscopy studies of stratum corneum lipid models. Acta Dermato Venereologica 208: 16-22.

15. Maynes R, Burgeson RE (2012) Structure and function of Collagen types. II. Maynes R, Burgeson RE, editors. Orlando, Florida: Elsevier.

16. Clark JA, Cheng JCY, Leung KS (1996) Mechanical properties of normal skin and hypertrophic scars. Burns 22:443-446.

17. Taylor SC (2002) Skin of color: Biology, structure, function, and implications for dermatologic disease. J Am Acad Dermatol 46: S41-62.

18. Fartasch M (1997) Epidermal barrier in disorders of the skin. Microsc Res and Tech 38: 361-372.

19. Nino M, Franzese A, Ruggiero Perrino N, Balato N (2012) The effect of obesity on skin disease and epidermal permeability barrier status in children. Pediatr Dermatol 29: 567-570.

20. Choudhury J, Mirshahi F, Murthy KS, Yager DR, Sanyal AJ (2006) Physiologic concentrations of leptin increase collagen production by nonimmortalized human hepatic stellate cells. Metabolism 55: 1317-1322.

21. Loden M, Olsson H, Axell T, Linde YW (1992) Friction, capacitance and transepidermal water loss (TEWL) in dry atopic and normal skin. Br J Dermatol 126: 137-141.
22. Escoffier C, de Rigal J, Rochefort A, Vasselet R, Leveque JL, et al. (1989) Age-Related Mechanical Properties of Human Skin: An In Vivo Study. J Invest Dermatol 93: 353-357.

23. Pawlaczyk M, Lelonkiewicz M, Wieczorowski M (2013) Age-dependent biomechanical properties of the skin. Pos dermatol Aller 30: 302-306.

24. Cork MJ, Danby SG, Vasilopoulos Y, Hadgraft J, Lane ME, et al. (2009) Epidermal barrier dysfunction in atopic dermatitis. J Invest Dermatol 129: 1892-1908.

25. Larios G, Gregoriou S, Rigopoulos D (2010) Letter: further options for treatment of hypertrophic scars and keloids. Dermatol Surg 36: 268-269.

26. Jichun Li J, Hongbin Liu H, Althoefer K, Seneviratne LD (2012) A stiffness probe based on force and vision sensing for soft tissue diagnosis. In: 2012 Annual International Conference of the IEEE Engineering in Medicine and Biology Society. IEEE 944-947.

27. Nomura T, Terashi H, Omori M, Sakurai A, Sunagawa T, et al. (2007) Lipid analysis of normal dermis and hypertrophic scars. Wound Repair and Regen 15: 833-837.

28. Desmoulière A, Chaponnier C, Gabbiani G (2005) Tissue repair, contraction, and the Myofibroblast. Wound repair and Regen 13: 7-12.

29. Suresh S (2007) Biomechanics and biophysics of cancer cells. Acta Materialia 55: 3989-4014.

30. Remmerbach TW, Wottawah F, Dietrich J, Lincoln B, Wittekind C, et al (2009) Oral cancer diagnosis by mechanical phenotyping. Cancer Res 69: 1728-1732.

31. Fomovsky GM, Thomopoulos S, Holmes JW (2010) Contribution of extracellular matrix to the mechanical properties of the heart. J Mol Cell Cardio 48: 490-496.

32. Souza RR de (2002) Aging of myocardial collagen. Biogerontology 3:325-335.

33. Chaturvedi RR, Herron T, Simmons R, Shore D, Kumar P, et al. (2010) Passive stiffness of myocardium from congenital heart disease and implications for diastole.Circulation 121: 979-988.

34. Yeh WC, Li PC, Jeng YM, Hsu HC, Kuo PL, et al. (2002) Elastic modulus measurements of human liver and correlation with pathology. Ultrasound Med Biol 28: 467-474.

35. Yin M, Talwalkar JA, Glaser KJ, Manduca A, Grimm RC, et al. (2007) Assessment of Hepatic Fibrosis With Magnetic Resonance Elastography. Clin Gastro Hepatol 5: 1207-13.e2.

36. Arda K, Ciledag N, Arıbas B (2013) Quantitative assessment of the elasticity values of liver with shear wave ultrasonographic elastography. Indian J Med Res137: 911-915.

37. Wellman PS, Howe RD, Dalton E, Kern KA (1999) Breast tissue stiffness in compression is correlated to histological diagnosis. J Biomech 1-15.

38. Wolfram D, Tzankov A, Pülzl P, Piza-Katzer H (2009) Hypertrophic scars and keloids--a review of their pathophysiology, risk factors, and therapeutic management. Dermatol Sur 35: 171-181.

39. Laviades C, Varo N, Fernandez J, Mayor G, Gil MJ, et al. (1998) Abnormalities of the extracellular degradation of collagen type I in essential hypertension. Circulation 98: 535-540.

40. Querejeta R, Lopez B, Gonzalez A, Sanchez E, Larman M, et al. (2004) Increased collagen type I synthesis in patients with heart failure of hypertensive origin: relation to myocardial fibrosis. Circulation 110: 1263-1268. 